\title{
OBSERVACIÓN DE LA CALIDAD DE LAS POLÍTICAS DE MOVILIDAD CICLISTAS DESDE LA PARTICIPACIÓN. Un estudio comparado de Madrid y Sevilla
}

\author{
Elisabeth LORENZI FERNÁNDEZ *; Carmen ACERO \\ *UNED, CIMAS (España) \\ elisabeth.lorenzi@gmail.com
}

\begin{abstract}
OBSERVATION QUALITY POLICY FROM BICYCLE MOBILITY PARTICIPATION. A comparative study of Madrid and Seville
\end{abstract}

Resumen: Presentamos resultados y reflexión sobre un estudio que aborda procesos de participación ciudadana en la implementación de los sistemas de movilidad ciclista y su interacción con factores que influyen en la variación de la ciclabilidad, comparando dos áreas metropolitanas españolas (Madrid y Sevilla) en el período 1990-2014. Esta investigación puede ser agrupada en torno a dos aspectos fundamentales que se distinguen y solapan a lo largo de la misma. Por una parte se pretende llevar a cabo un estudio longitudinal y comparativo de los procesos de implementación versados en la movilidad sostenible en las ciudades de Madrid y Sevilla, viendo, del mismo modo, cuáles han sido las mutuas afecciones entre: movimientos probici, políticas públicas, medio ambiente y configuraciones sociales. Nuestro proyecto aborda estas cuestiones poniendo especial foco empírico en el discurso y las prácticas de las instituciones políticas y de los movimientos sociales para analizar su interacción y su impacto en la ciclabilidad de las ciudades de Madrid y Sevilla. A nivel metodológico nuestra investigación ha recogido discursos a través entrevistas en profundidad, observación participante y talleres de devolución y auto diagnóstico. Entendemos que la posibilidad que las metodologías participativas brindan a la hora de exponer y tratar en común los diferentes puntos de vista, inducen a un mayor acercamiento a las múltiples caras que la realidad tiene, logrando, a su vez, una mayor adecuación entre teoría y realidad dada.

\footnotetext{
Abstract: We present results of a study and reflection about our work studying the mutual influence among the citizenships initiatives and the policies on the field of biking mobility in Spain comparing two spanish metropolitan areas, Madrid and Sevilla (1990-2014). Many operative dimensions have been attempted in order to improve sustainable urban mobility policies. We need to deepen into some aspects: A multi-level and transitional approach will be used: a) cycling policies are conceived as a result of four levels of action mutually interacting (urban policies, pro-bike urban movements, urban environment, and social patterns); b) transition processes from automobility, regimes are due to specific social and political factors which can ameliorate or hinder them. Finally, these multi-level interactions and transitional factors will be verified through a participatory action-research methodology which can open spaces for public deliberation on the issue of urban Our project addresses these issues with special focus on empirical discourse and practices of political institutions and social movements to analyze their interaction and their impact on the cyclability of cities. We adopt a participatory approach where citizen participation of multiple social agents will be taken into account as both an object of research and a means of debating and promoting bicycling policies. Comparison between two cities with different achievements in terms of cyclability can provide a better understanding of the role of citizen participation within sustainable urban mobility policies. Methodologically our research has collected speeches through in-depth interviews, participant observation and workshops return and self diagnosis. We understand that the possibility that participatory methodologies provide when present and discuss together the different points of view, induce closer to the many faces that reality has, achieving, in turn, a better match between theory and current reality.
}

Palabras clave: Ciclismo urbano, movilidad sostenible, participación ciudadana, estudio comparativo, investigación acción participación. Urban Cyclism, sustainable mobility, citizen participation, comparative study, action research participation. 


\section{Bicipart: un estudio comparado de las políticas de movilidad}

El proyecto de Bicipart fue un estudio que analizaba las interacciones entre Políticas Públicas e iniciativas sociales en materia de movilidad ciclista, observando cómo esta interacción dejaba huellas en la conformación de la ciudad: "Procesos de Participación Ciudadana en la Implementación de Sistemas de Movilidad Urbana Sostenible" (Bicipart- CIMAS, 2015). En este proyecto comparamos dos ciudades, Madrid y Sevilla y el trabajo de investigación fue llevado a cabo por un equipo interdisciplinar en el campo de las ciencias sociales.

El análisis de la movilidad ciclista es un fenómeno relativamente reciente en España. Aún así, la realidad sevillana ha sido ampliamente analizada por el rápido despegue del uso de la bicicleta urbana en pocos años. Aunque la producción científica en castellano en este ámbito es incipiente, consideramos que nuestro estudio puede aportar una importante innovación en el creciente campo de la movilidad ciclista a nivel internacional por su foco de atención. Hasta la actualidad ha habido una gran producción desde diversas disciplinas en el análisis de los diferentes factores que influyen en el uso de la bicicleta. Pocos de estos análisis centran su atención en la participación ciudadana como factor clave del desarrollo de la movilidad ciclista y además, desde una perspectiva cualitativa.

Nuestra perspectiva no es casual y viene espoleada por el crecimiento de la visibilidad y protagonismo de las iniciativas sociales en la promoción del ciclismo urbano en un contexto mundial de crisis energética y ambiental y en un marco paneuropeo de promoción de políticas de movilidad sostenible, donde la "cultura de la bicicleta" es observada como factor de influencia en la ciclabilidad de las ciudades y desde su producción en el seno de las interacciones sociales. Esta corriente de producción teórica y empírica parece ir parejo al auge de iniciativas de promoción del uso de la bicicleta desde la esfera social.

Las referencias empíricas de los autores de estos textos hacen referencia a la acción colectiva y movilizadora (Carlsson 2001; Furness 2010, Lorenzi 2010) a actividades de ocio como marchas ciclistas (Aldred, 2010) y a prácticas de bike commuting (ir en bici al trabajo) (Skinner and Rosen 2007) y a la relación entre las referencias y las percepciones (Spinney, 2007). Sus pautas de reflexión conectan con los conceptos de cultura, identidad y cambio social y con las herramientas teóricas de la Sociología, la Antropología y de los estudios sobre Movimientos Sociales. Esto último es importante ya que observa prácticas que se inscriben en la promoción del uso de la bicicleta y por tanto en la promoción del cambio social.

Por otro lado, la cuestión de la identidad cobra especial relevancia, no sólo para comprender los procesos de difusión del ciclismo, sino como clave estratégica de las iniciativas de la sociedad civil de promoción ciclista: revertir el estigma del ciclista, generar sentimiento de pertenencia y redes de apoyo mutuo y una relación diferente con el espacio público y el concepto de la ciudad. Este es el salto que nos permite trabajar los procesos de cambio social y el papel de la ciudadanía. En estos estudios la bicicleta no es simplemente una opción que necesita ser ampliada, sino que es un instrumento de transformación del concepto mismo de la ciudad. Así Rachel Aldred (2010) nos señala cómo la bicicleta en sí misma, está cargada de potenciales que sostiene los valores ciudadanos: autonomía personal y sostenible, localización del arraigo, y apertura hacia la comunidad y respeto al medio La bicicleta en un dispositivo de cambio no solo del medio ambiente sino de la recuperación de un modo de vida comunitario que el aumento del uso del coche ha desarticulado. Alfonso Sanz (2008) nos recuerda que el tráfico motorizado socava las redes de comunicación vecinal. En este sentido referencias a la recuperación del espacio público como el espacio de la ciudadanía y de la bicicleta son abundantes. David Horton (2007) nos señala cómo el coche es una extensión del hogar extendido al espacio público ante lo cual el ciclista vive bajo el escrutinio de los demás conductores. Rachel Aldred (2010) nos señala el potencial comu- 
nitario por la recuperación de las calles como un espacio de socialización y Chris Carlsson (2001) señala cómo la mismas masa de ciclistas se convierte en un lugar de socialización, una "comunidad" en movimiento mientras que se hace realidad en el propio momento lo que se reclama para un futuro inmediato.

Por otro lado, el ciclismo urbano se ha convertido en un repertorio de lucha, una práctica que se ha difundido a numerosas ciudades del mundo y en cuyo contexto la bicicleta se convierte en algo más que un modo de transporte, sino en un caballo de batalla (Lorenzi, 2010). Así lo señala Chriss Carlsson y los testimonios que edita en su libro "Critical Mass. Bicycling's Defiant Celebration" cuando los protagonistas de cada testimonio hablan del surgimiento del movimiento en sus propias ciudades y como las formas reconocidas mundialmente son reproducidas con los elementos locales de cada ciudad.

A nivel general, nuestro estudio aborda las prácticas ciclistas como resultado contingente de una combinación de cuatro niveles de acción y condicionamiento poniendo especial hincapié en las dos primeras como principal foco de recogida y análisis de discurso: políticas urbanas, iniciativas pro-bici, cualidades de cada entorno urbano y los conocimientos, hábitos adquiridos; actitudes y percepciones ante la cuestión de la movilidad

Elegimos Madrid y Sevilla como ciudades a comparar por las siguientes razones. Ambas son ciudades definidas "en transición ciclista" (aunque todas las ciudades españolas lo son) y la participación ciudadana ha jugado un papel importante y diferencial en su crecimiento: En Sevilla, los presupuestos participativos definieron la inversión en infraestructura viaria ciclista que tuvo como resultado la construcción intensiva de más de $140 \mathrm{~km}$ de carril bici durante el ejercicio municipal de 2006-2010. En Madrid, las iniciativas pro-bici ciudadanas espolearon el rápido crecimiento del número de ciclistas en las calles de la ciudad, aunque con tímidos apoyos desde el gobierno municipal.

Nuestro propósito a lo largo de este artículo es reflexionar sobre las dimensiones conceptuales de Movilidad a la par que mostramos sus consecuencias a nivel de las prácticas políticas, tanto de las instituciones de gobierno como de la iniciativa ciudadana. Más adelante, mostraremos cual es la metodología que negociamos como equipo multidisciplinar para abordar estas dimensiones, reflexionando sobre sus límites y potenciales. Finalmente mostraremos como esta metodología nos llevó a los principales hallazgos empíricos de nuestro estudio.

\section{La política de la bicicleta}

Hoy en día es ineludible pensar en la movilidad en términos políticos. Esto se hace especialmente evidente cuando notamos que el término Movilidad ya aparece en el nombre de las políticas, los departamentos, las consejerías, planes y programas, cuando hace algunos años era la palabra Tráfico la que cumplía este lugar. Esto implica una carga conceptual donde se reconoce que el movimiento de las personas es más que una gestión de recorridos y de cargas.

Un análisis bibliográfico de la producción científica de los últimos 15 años en esta materia (Bicipart, 2015; 6-10) ha evidenciado que las propias iniciativas sociales han ido ensanchando y produciendo con su práctica el concepto mismo de Movilidad y todo lo que ello implica. Si en un principio se hablaba en términos de transporte, de tráfico, de circulación..., la Movilidad es un campo que incorpora la percepción, la actitud, los hábitos, los derechos... En los últimas dos décadas, las iniciativas pro-bici, se han ocupado de reivindicar ante las autoridades un modelaje diferente de las infraestructuras urbanas para aumentar la presencia del ciclista en la calzada y el espacio público, pero también han trabajado hacia los ciudadanos y la sociedad en general, animándoles a un cambio activo en sus prácticas de movilidad. Por "Iniciativa pro-bici" entendemos toda acción o plataforma pro-activa y participativa cuyo fin principal es promover el uso de la bicicleta y abarcaría tanto formas de 
articulación asociativa, como eventos puntuales, entidades formales como informales, con adscripciones puntuales como continuas.

\section{Movilidad ciclista en Madrid y Sevilla}

En el momento en que cerramos el estudio (2014), los datos urbanísticos sobre movilidad de ambas ciudades nos mostraban que en Madrid el número medio de viajes era de 2,6 por habitante al igual que en Sevilla. El reparto modal de Madrid era de un $31 \%$ a pie, $0,3 \%$ bicicleta, $34 \%$ Transporte público y $35 \%$ en coche o moto. En Sevilla el reparto modal era de un $37 \%$ a pie, $3 \%$ en bicicleta, $20 \%$ en transporte público y un $40 \%$ en coche o moto Monzón et al. 2013). Sevilla presentaba una mayor participación de los modos no motorizados, aunque en Madrid había un menor uso del vehículo privado motorizados.

En los últimos años, Madrid y Sevilla muestran un crecimiento urbano con tendencias a la dispersión, es decir, a crecer en densidades poblacionales más bajas que en el área metropolitana, registrándose un descenso en la densidad del 10\%. Esta dispersión urbana ha traído como consecuencia un mayor protagonismo de la movilidad motorizada en ambas áreas metropolitanas, con crecimientos del 50\%, e indicios de una mayor dependencia del coche (Monzón y de la Hoz, 2011). En lo que se refiere a la movilidad no motorizada esta se sitúa en un 33,5\% para Madrid y un 40,8\% para Sevilla.

Atendiendo específicamente al uso de la bicicleta en ambas ciudades se pueden constatar incrementos significativos. Madrid va duplicando su uso año por año, por ejemplo en 2013 el incremento fue de un 0,1\% a un 0,6\% del reparto modal (Ayuntamiento de Madrid 2013), mientras que Sevilla tuvo un aumento más espectacular del $0,5 \%$ a un $3 \%$ en cuatro años (NOSDO, 2007; NOSDO, 2010). Prestando atención al origen de esos nuevos viajes que se hacen en bicicleta, sería deseable que el trasvase modal se produjera desde la movilidad motorizada, especialmente desde el coche, pero algunos indicadores indican que muchos viajes provienen de viajes anteriormente realizados a pie como consecuencias de distancias de viajes mayores (Fernández Heredia y Rondinella 2012).

\section{El análisis dinámico y estático del sistema de movilidad de una ciudad. La partici- pación como factor de estudio y las herramientas participativas introducidas en la investigación.}

Como se desprende de nuestro objeto de estudio y la forma en que lo presentamos, estudiar qué cuestiones propician el desarrollo de la movilidad ciclista en una ciudad implica, por un lado considerar que el sistema de la movilidad puede ser analizado de forma estática y por otro de forma dinámica. El nivel estático de evaluación del sistema de transporte en su relación con la movilidad ciclista para ciudades en transición se puede definir a través de cuatro niveles:

- Las Políticas urbanas: de movilidad en general y de movilidad ciclista en particular, aunque también las de ordenación territorial y urbanística.

- Los Movimientos sociales relacionados con la bicicleta: asociaciones, colectivos y "masas críticas", sobre todo.

- Las Cualidades singulares de cada entorno urbano construido y medioambiental (orografía, climatología, red viaria, infraestructuras y configuración urbana funcional).

- Las Configuraciones sociales: conocimientos y hábitos adquiridos; actitudes y percepciones ante la seguridad, la comodidad y la eficiencia; prácticas y redes de difusión; influencia del género, la edad, la etnia y la clase social).

Comprender las interacciones entre los cuatro niveles señalados, ha sido objeto de nues- 
tra investigación, y requiere de técnicas que expliquen los procesos de transformación social en torno a la bicicleta como las empleadas en Sociología y Antropología. De ahí que nuestro planteamiento no sea otro que, partiendo de estos cuatro vectores y del saber acumulado en la ciencia del transporte, combinarlos con metodologías destinadas a identificar y explicar estas interacciones. Las propias técnicas utilizadas impiden extender las conclusiones más allá que al análisis comparado de las ciudades seleccionadas: en nuestro caso Madrid y Sevilla.

La caracterización de los cuatros vectores propuestos para dibujar el mapa del sistema de transporte en referencia a la movilidad ciclista se puede resolver parcialmente por el acceso a fuentes de datos directas y estudios previos. El nivel dinámico requiere de otro tipo de técnicas de naturaleza cualitativa.

A la hora de abordar la parte dinámica de nuestro estudio hemos desarrollado diferentes metodologías cualitativas entre las que dimos un peso especial a las metodologías participativas. Así, por un lado, la participación ciudadana, fue considerada de forma central en la investigación como objeto de estudio a la vez que como medio de investigación sociológica y como forma de intervención social, aunque no tanto como hubiera sido deseable. Por razones de diversa índole (sobre todo de presupuesto), no se pudo llevar a cabo el ciclo completo de una investigación participativa, como era nuestra intención inicial. Pero sí que introdujimos algunas herramientas propias de las metodologías participativas, pero de ciclo más corto.

Entre ellas, el sociograma que mediante la observación y la contextualización, muestra las relaciones reticulares existentes en una situación dada, en nuestro caso las relaciones entre los actores relacionados con la movilidad ciclista de ambas ciudades: Madrid y Sevilla. Usando esta técnica se logra situar las relaciones y grupos existentes, de forma que se puede actuar tanto grupal como individualmente sobre las mismas, dando una visión de conjunto del marco estudiado. Con el sociograma también se tiene acceso de una forma pormenorizada y ajustada a la realidad de las características estructurales que, en parte, serán las que a su vez estructuran la variabilidad discursiva en torno a la problemática de estudio. (Red Cimas, VVAA 72: 2016) Por ello fue una herramienta imprescindible a la hora de elaborar la muestra estructural o significativa de las personas entrevistadas. Así mismo, el sociograma es una herramienta de gran utilidad a la hora de generar dispositivos de participación en y a partir de los diferentes talleres.

Partiendo del sociograma y del trabajo previo llevado a cabo, se preparó un guión de las entrevistas en profundidad. Las entrevistas eran diferentes en función eje discursivoestructural en el que el entrevistado participaba. Este guión se ampliaba y adaptaba cuando encontrábamos posiciones discursivas existentes dentro del campo que todavía no habían sido cubiertas en las entrevistas.

Partiendo de la información obtenida del sociograma se ha llevado a término una serie de entrevistas tanto a "expertos temáticos" como a "expertos convivenciales". Estas entrevistas han sido realizadas a los sujetos más representativos, tanto de la Administración, de los movimientos pro-bici, mundo empresarial, académico como a técnicos cualificados en la materia. El número total fué de 24 entrevistas, doce por ciudad. Las entrevistas fueron transcritas para poder ser analizadas y registradas. Una vez transcritas, la información fue analizada por bloques de posición en el sociograma y por ciudad para, luego ser contrastadas en un análisis de equipo

Por último los talleres de devolución y autodiagnóstico en los que se aplicaron varias técnicas participativas como los multilemas, flujogramas y árbol de problemas.

Con el multilema se intenta romper con las lógicas dicotómicas a la hora de plantear un problema, a favor o en contra, que por otro lado son las posiciones más fáciles de encontrar a la hora de analizar un problema.. Si queremos superar estas situaciones antagónicas tenemos que abrir nuevos planos de interpretación, cuestionar la pregunta inicial que nos 
está llevando a esta situación de bloqueo. La técnica del tetralema/multilema consiste en ir agrupando las frases a lo largo de un eje imaginario, en el que se representan las diferentes posturas, marcando ejes dominantes y posiciones intermedias. Esta disposición nos permite incorporar también aquellas posturas no dominantes, las que aportan matices o aquellas que aportan una mayor creatividad al análisis colectivo.(Villasante Tomás R 92:2004)

Se presentaron dos multilemas, uno que expusiera parte de la historia, desarrollo y evolución de la movilidad urbana en bicicleta en cada ciudad, y otro en el que se presentaron los debates contenidos en la misma, las herramientas, métodos y modelos implementados.

La otra técnica empleada fue el Flujograma, con la que se mostraron los puntos centrales y controversias en la aproximación a las problemáticas relacionadas con la movilidad y la bicicleta para que los participantes establecieran las relaciones causales entre ellas. El flujograma es un ejercicio que nos permite establecer colectivamente la valoración de las relaciones causa-efecto entre los elementos que se recogen en las frases seleccionadas. Con ello se pueden priorizar los temas más destacados para abordar colectivamente sus posibles soluciones, así como identificar a los actores responsables y buscar estrategias de mejora (Red Cimas 102:2016) Consiste en elaborar colectivamente un gráfico en el que se visualicen las relaciones causa-efecto entre los diversos elementos relacionados con el tema objeto de debate, para establecer los "nudos críticos" o principales factores a resolver. Esto se consigue contabilizando el número de "entradas" y "salidas" de cada elemento como causa o consecuencia.

Por último completamos esta sesión con la técnica del "Árbol de problemas" que nos sirvió a partir de las frases trabajadas en la dinámica anterior, para identificar los síntomas que dan cuenta de un problema y relacionarlos con el análisis de sus causas inmediatas y sus causas profundas. La representación gráfica de estas relaciones permitió visualizar colectivamente con claridad la relación entre las causas profundas o de raíz, las causas inmediatas así como la visibilidad o síntomas de los problemas de la movilidad en Madrid (Fig.1.).

\section{El análisis comparativo. Potenciales y dificultades}

Entender el proceso de cambio de paradigma a una movilidad sostenible de mano de un mayor uso de la bicicleta pasa por estudiar las ciudades que están en este proceso, en mayor o menor grado. Para ello dejamos fuera de nuestra propuesta las ciudades con un uso normalizado y consolidado de la bicicleta o aquellas donde este modo sigue siendo residual y no se perciben evolución alguna en este sentido.

La comparación de las dos ciudades elegidas en este estudio iba dirigida a poder extraer conclusiones acerca de los factores y procesos de ejecución de políticas probici, porque ambas se han distinguido de forma diferente por la implicación ciudadana en las mejoras de ciclabilidad de sus calles a lo largo de las dos últimas décadas. Aunque nuestro planteamiento inicial incluía desarrollar esta comparativa en al menos cuatro casos de ciudades españolas, finalmente, y por una cuestión presupuestaria, nos vimos en la necesidad de ceñirnos a dos casos.

Numeramos algunas las potencialidades y debilidades que consideramos más relevantes de la comparación entre ambas ciudades. En este sentido, ha sido posible poder modelizar o sintetizar los dos casos de promoción de políticas probici, pudiendo analizar todos los elementos que nos propusimos.

- Potencialidades: Tanto Sevilla como Madrid son ciudades en transición ciclista con desarrollos en movilidad sostenible muy diferentes. Por un lado, Madrid ha estado más volcada en el desarrollo de iniciativas culturales y pedagógicas. Por otro lado, en Sevilla se ha realizado con mayor profusión el desarrollo de infraestructuras ciclistas. También ha sido fundamental profundizar en las dos formas de gestionar e incluir la participación ciudadana en temas de movilidad ciclista por parte de ambos gobiernos municipales, uno vinculante y 


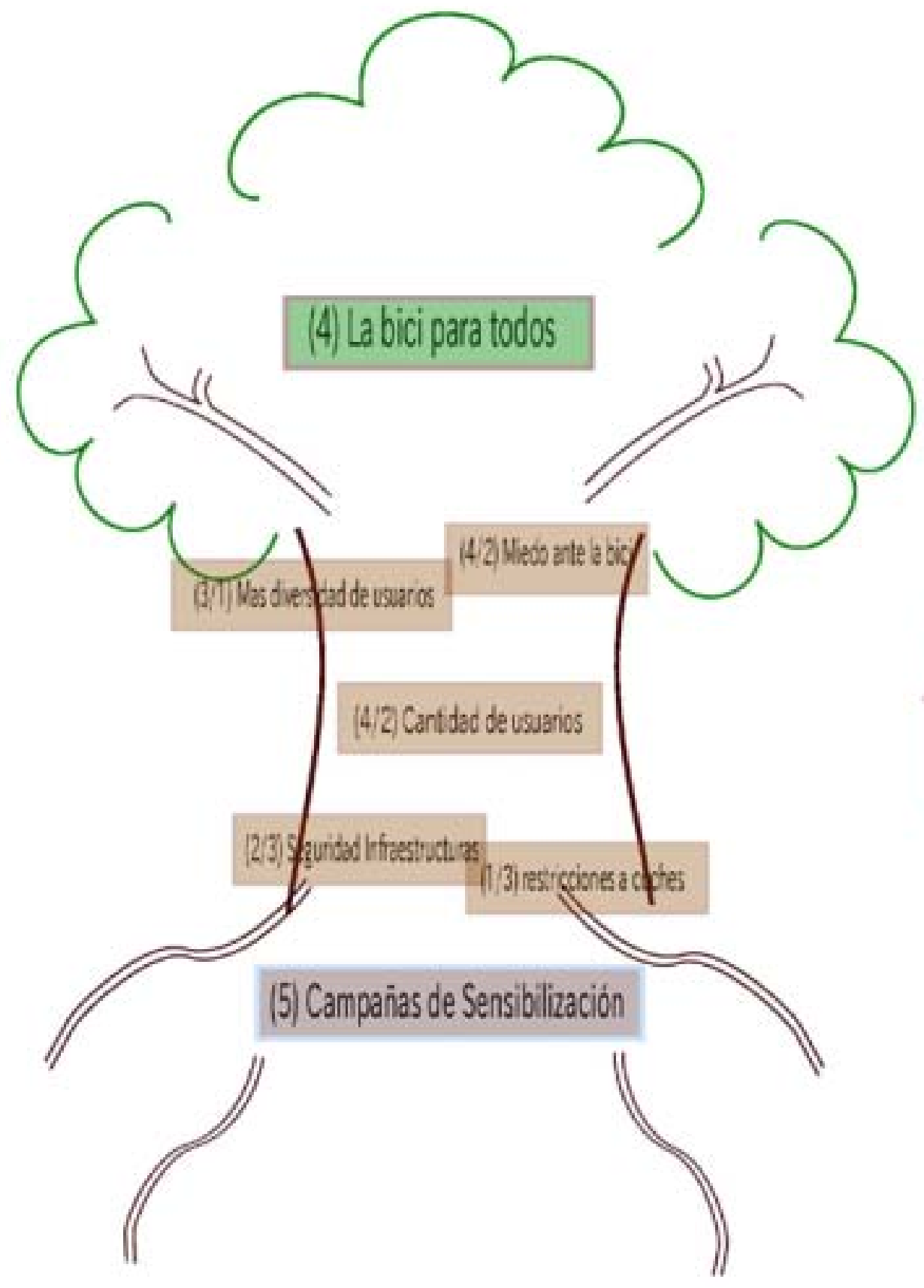

Fig.1. 
que desarrolla mecanismos para seguir contando con la participación ciudadana (Sevilla) y el otro modelo únicamente consultivo (Madrid).

- Dificultades: Por contra, el trabajo de campo fue desigual al existir una mayor dificultad para entrar en contacto y conocer las redes en Sevilla, pues todo el equipo investigador pertenecía a Madrid, con trabajos de investigación en esta ciudad ya previos.

Sin embargo en relación a la producción de datos estadísticos había mucha más referencias en torno a la ciudad de Sevilla que a la de Madrid. En este sentido, toda la ventaja que lleva Sevilla a Madrid en su implantación de políticas ciclistas ha favorecido el desarrollo de una especial atención de bibliografía en torno al estudio de su realidad.

\section{Resultados empíricos: los discursos entorno a la política de la movilidad}

La recogida de datos produjo principalmente un cruce de discursos entre protagonista de las iniciativas pro-bici, agentes políticos y agentes técnicos en materia de movilidad ciclista

En Madrid, se aprecia que si bien existen políticas encaminadas al desarrollo de la movilidad ciclista, éstas no habían sido centrales en el marco de las políticas de movilidad sostenible. Por otro lado, su desarrollo y aplicación no había seguido claramente unos parámetros de una política de medio o largo plazo. Sus objetivos e instrumentos parecían poco claros y sujetos a frenazos y tirones. Los "tirones" más fuertes venían provocados por las directivas europeas dirigidas a reducir el nivel de contaminación atmosférica de la ciudad. Los "frenazos" más destacados están influidos por la percepción de que apostar por modelos ciclistas va en contra de un modelo democrático de la movilidad y de accesibilidad (se valora que la bicicleta no es una opción para todos los ciudadanos) y que las políticas ciclistas podrían resultar impopulares ya que van en detrimento del espacio concedido al coche.

En lo que se refiere a las iniciativas ciudadanas, partiendo de cierto tronco común, se han desplegado en pocos años en una gran diversidad de referentes ideologías y prácticas, pero con cierta tendencia a confluir en el objetivo común, la promoción de la bicicleta como modo de transporte en Madrid. Este amplio rango ha ayudado a que el ciclismo urbano se expandiera tímidamente, pero con constancia. Estas iniciativas, dirigen su mensaje principalmente a la sociedad, incidiendo en su percepción del entorno urbano y de sus hábitos, aunque algunas de estas iniciativas optan también por enfocar su mensaje a la administración municipal dentro de los cauces que se abren para ello.

Aunque la administración se abre cada vez más a la participación ciudadana en el diseño o valoración de sus políticas, el papel de los ciudadanos hasta ahora ha sido consultivo. La inversión de energía de estos colectivos en estos procesos, no ha ido en proporción directa a los resultados que han obtenido, pero percibían que esta interacción influye poco a poco en el discurso y en las formas de diseñar las vías ciclistas por parte de un Ayuntamiento que cada vez apostaba menos por modelo segregacionistas, cambio que podría estar también motivado por razones económicas. Por otro lado, las políticas de sensibilización hacia la sociedad en general ha sido el marco en el que mayormente se propiciado buenas prácticas de colaboración entre iniciativas por-bici e instituciones

En Sevilla se daba una percepción compartida de que las políticas públicas habían apostado por la bicicleta de forma decidida y que el punto de partida de las políticas ciclistas reales fueron los presupuestos participativos de 2004. La apuesta decidida de Sevilla por la movilidad ciclista se explicaba con tres claves: uso masivo de los movimientos sociales de los canales abiertos de participación, que su vez generó un respaldo a la administración con activa voluntad política y, finalmente, un alto uso de las infraestructuras ciclistas implementadas, refrenda las medidas adoptadas. Se percibía un amplio consenso respecto a que la alineación de movimientos sociales con las instituciones políticas ha permitido un mayor alcance de las políticas de movilidad ciclista. Todo esto contrastaba con cierto parálisis de decisiones políticas en Madrid, producida por el miedo al rechazo de la opinión pública a 
determinadas medidas de políticas ciclistas.

Este respaldo social a las instituciones ha generado un marco favorable para que diferentes políticas ciclistas se hayan desarrollado en la ciudad: implementación de un sistema de bicicletas públicas, puesta en servicio de una red ciclista extensa y conexa, medidas de educación y sensibilización, cambios en las ordenanzas de tráfico, etc. Tanto políticos, técnicos y colectivos ciclistas inciden en las sinergias que se han producido fruto de la colaboración, especialmente por la participación de los colectivos ciclistas en el diseño de las medidas tomadas.

En Sevilla se destacaba el papel de la participación pública en el avance de las políticas ciclistas por diversos factores: beneficios del respaldo de la opinión pública, obtener el aval de la ciudadanía y sus consecuencias en la legitimidad de las decisiones, incorporación del bagaje técnico de los movimientos sociales en determinados campos de innovación donde la administración puede estar menos preparada y producir una normativa más adaptada a las casuísticas reales.

\section{La bicicleta, palanca para estudios transdisciplinares}

Una de los potenciales que ofrece trabajar en el campo de la movilidad ciclista es la absoluta necesidad de entrar en diálogo con otras disciplinas. El equipo de Bicipart contaba con profesionales de la sociología con experiencia en metodologías de investigación participativa, un urbanista y una antropóloga.

Al mismo tiempo, a la hora de trabajar la bibliografía de referencia, tuvimos que entrar a jugar con aportaciones de campos muy diversos con el común denominador de la movilidad ciclista (psicología, ciencias políticas, filosofía, geografía...) En este sentido nos dimos cuenta que la producción bibliográfica en este campo tradicionalmente había estado muy ligada a estudios cuantitativas y que poco a poco, visiones más cualitativas habían ido ocupando su espacio y ampliando el concepto de Movilidad.

En cuanto a la perspectiva teórica y metodología de trabajo, el contar con un equipo diverso, nos permitió establecer una visión multifocal de los condicionantes de la movilidad, Sin embargo, la limitación de los fondos influyó que ante la multiplicidad de herramientas metodológicas, nos apoyáramos en aquellas que suponían menor inversión de recursos. Por tanto focalizamos la mayor parte de nuestros recursos humanos en la aplicación de metodologías cualitativas de corto plazo, como las entrevistas y talleres de participación. Datos cuantitativos se usaron, pero no se produjeron. Se aplicó observación de campo, pero se dio de forma más esporádica y dependiendo de la cercanía de las fuentes. Al estar el equipo de trabajo ubicado en Madrid, propició que los hubiera mayor riqueza de observaciones participadas de esta ciudad que de Sevilla, y que se diera mayor fluidez con los informantes y con los contextos de participación de la bici, cosa que redundaba a su vez con más elementos de contraste y referencias a la hora de la triangulación de datos y facilidad a la hora de convocar los talleres participativos y en los resultados de los mismos

La diversidad de perspectivas, junto al reparto de tareas del equipo por ciudades, también supuso una intensa inversión de tiempo en reuniones internas en las que debíamos consensuar los términos y epígrafes a usar, el modo de sistematizar la información que fuera satisfactorio con los objetivos del estudio y con el bagaje metodológico de cada perfil. Este proceso no fue sencillo, pero se encontró cierto equilibrio entre renuncias, enriquecimiento transdisciplinar y las posibilidades dadas por los recursos que teníamos a disposición. Podríamos decir que esta negociación continua y la necesidad de adaptación, supuso también una fuente de innovación metodológica, también propiciado por la oportunidad que brinda la observación de la movilidad ciclista y todos los aspectos que son necesarios de observar. 


\section{Conclusiones}

Bicipart ha sido una experiencia que ha permitido evaluar la calidad de las políticas ciclistas en relación a su grado de participación en las mismas. Nuestro proyecto entiende que cuanta mayor es la participación en las políticas mayor es su calidad y ajuste a las necesidades del territorio y de sus habitantes.

Esta imbricación de agentes depende en gran medida de la voluntad política, del tipo de procesos participativos que se generan, del background técnico de los especialistas que trabajan para la administración y del repertorio de participación de las iniciativas pro-bici.

La participación ciudadana es importante por varias razones. La práctica del ciclismo urbano genera conocimiento. Como dice Lucio Gil $(2012 ; 6)$ La calidad de la respuesta a los desafíos de movilidad estar determinada por la capacidad de gestionar el conocimiento valioso pero disperso que se encuentra potencialmente en el tejido social y profesional de la Ciudad. Por otro lado, una política participada genera legitimidad en la aplicación de las políticas pro-bici, esto es importante en la aplicación de unas políticas que a nivel general, despierta simpatía en la sociedad, pero no se percibe aún como una solución de movilidad universal.

Por otro lado, las iniciativas pro-bici están más dispuestas a participar en los procesos que se abren desde las instituciones cuando perciben que el esfuerzo invertido va ser proporcional a los resultados. Para que esto sea así, es necesario que estos procesos tienden a ser más vinculantes que consultivos.

Nuestros casos de estudio mostraron que en Madrid, los resultados del encuentro entre ciudadanía e instituciones, en materia de ciclismo, ha sido mucho más fluida cuando se trataba de políticas y planes de sensibilización hacia la sociedad. Cuando en cambio se trataba de influir en la gestión de las infraestructuras viales, había influencia, pero no era proporcional al esfuerzo que invirtieron los colectivos. Los colectivos señalaron que una de las razones es la falta de voluntad política. Coinciden con las instituciones la percepción de la sociedad no está preparada y que necesita muchas actuaciones en materia de sensibilización

En cambio, en Sevilla fue el proceso de los presupuestos participativos por barrios lo que definió la inversión municipal en vías ciclistas y fue posteriormente una fuente principal de legitimación ante la oposición de ciertos colectivos ante el avance de las obras de infraestructura por toda la ciudad.

No obstante comprobamos que los avances conseguidos en materia de movilidad como en cualquier política pública está expuesta a sufrir cambios y retrocesos condicionados por el reemplazo de los gobiernos municipales. Por ejemplo, la situación en Sevilla ha ido cambiando a la par que lo hizo el signo del gobierno municipal en el año 2011. En esta legislatura se hace una política de "vuelta atrás" hacia la promoción del automóvil (permitiendo el acceso en coche al centro de la ciudad y ampliando las opciones de aparcamiento). Además se eliminó la convocatoria de la Mesa Cívica de la Bicicleta que aglutina a los diferentes actores relacionados con la promoción de la bici en la ciudad.

Aunque la participación ciudadana en el diseño de políticas públicas está siendo cada vez más valorada, sobre todo desde los nuevos gobiernos de corte municipalista surgidos tras las elecciones de 2015. Su introducción suscitan una serie de cuestiones importantes que hemos recogimos en nuestro estudio.

A veces ocurre que el conocimiento técnico ciudadano/activista sobre un asunto es más avanzado que el de la propia institución, esto genera que ésta cuente con el asesoramiento formal o informal de entidades sociales en el desarrollo de medidas. También comprobamos cómo introducir la participación ciudadana impulsa la creatividad e innovación social frente a los diferentes problemas urbanos.

En el caso de Madrid, permitió el avance hacia medidas integradoras de la bici en la 
calzada, pero además existe todo una producción de herramientas muy útiles para la promoción de la bici en Madrid, como son los mapas de calles tranquilas que en 2013 elaboró los editores del blog Muévete en bici por Madrid. Por lo tanto, la proactividad de algunos colectivos comprometidos ha llegado a generar una mayor versatilidad y eficacia en la capacidad de dar respuestas a demandas ciudadanas que desde la propia administración. Enumeramos algunos ejemplos como son la creación de herramientas virtuales, mejor accesibilidad a información gracias una mayor presencia en redes sociales, generación de debate en torno a temáticas controvertidas en foros, etc.

Es necesario combinar diferentes niveles de participación atendiendo a la diversidad de agentes sociales y a su disposición en el territorio. Abrir canales de participación formales, bien pensados y vinculantes en áreas de interés para la ciudadanía, como la movilidad, aumenta la calidad democrática de la vida en común y son una fuente fundamental de legitimidad de las políticas y por tanto de éxito. Por vinculante entendemos que la toma de decisiones no es exclusivamente institucional sino que la ciudadanía tiene acceso a ella (Ej: en Sevilla, los presupuestos participativos fueron un impulsor de las políticas ciclistas al resultar la medida de carril bici como elegida en 18 de las 21 asambleas territoriales). La participación no debe reducirse a los agentes sociales o usuarios que son especialmente activos o que están organizados, hay que aumentar al resto de capas y organizar procesos que permitan llegar a más gente afectada Y por último, es necesario evaluar de forma continua y participada por los diferentes agentes interesados las políticas de movilidad llevadas y no llevadas a cabo.

Por último, no todas las iniciativas pro-bici dirigen su mensaje hacia el mismo interlocutor. Las hay que se dirigen a la sociedad, a las entidades públicas, al tejido empresarial o a varias a la vez. Entender esta diversidad es importante para diseñar formas de participación inclusivas y que no impliquen un desgaste del tejido social

Para llegar a estas conclusiones ha sido crucial la devolución que hicimos a nuestros entrevistados sobre nuestras proposiciones preliminares. Esto nos ha permitido contrastar los discursos que habíamos analizado a partir de las entrevistas, visualizar nuevas conexiones y establecer un rango de propuestas. En estos talleres participaron tanto miembros de iniciativas pro-bici, técnicos y políticos municipales. Esta interlocución ha sido también una forma que ha permitido afinar y concretar las propuestas metodológicas del equipo que ya venían condicionadas por el presupuesto y por la confluencia de múltiples perfiles de investigación.

\section{Bibliografía}

Aldred, Rachel (2010) “On the outside. Constructing cycling citizenship”, en Social and Cultural geography, Vol 11, $\mathrm{N}^{\circ} 1$. London: Routledge.

BICIPART (2015) Procesos de Participación Ciudadana en la Implementación de Sistemas de Movilidad Urbana Sostenible (Bicipart)._http://ciclopart.redcimas.org/wp-content/ uploads/2012/08/Informe-FINAL BICIPART.pdf

Carlsson, Chris (2002) Critical Mass: Bycicling's Defiant Celebration.Oakland: AK Press.

Carlsson, Chris (2008) Nowtopia: How Pirate Programmers, Outlaw Bicyclists, and Vacant-Lot Gardeners are Inventing the Future Today! Oakland:A K Press

Horton, David (2010) "Fear of Cycling" in Cycling and Society. Ashgate eBook. London

Lorenzi, Elísabeth (2010) "Centro social en movimiento.Los talleres de auto-reparación de bicicletas en los espacios autogestionados", en Mario Domínguez, Miguel Martínez y Elísabeth Lorenzi, Okupaciones en movimiento. Derivas, estrategias y prácticas. Madrid: Tierra de Nadie

Lucio Gil, Antonio (2012) “¿La movilidad de Madrid puede tener remedio?” en Revista Ambient@ n⿳ 100, 2012-09-01) Ministerio de Alimentación, Agricultura y Medio Ambiente

Monzón de Cáceres, Andrés; de la Hoz Sánchez, Daniel (2011) Efectos sobre la movilidad dinámica territorial de Madrid. Urban, 58-71.

NOSDO (2010) Estudio sobre el uso de la bicicleta en la ciudad de Sevilla. 
RED CIMAS (2015) Metodologías Participativas, Sociopraxis para la creatividad social. Madrid: Dextra editorial.

Sanz, Alfonso (2008) Calmar el tráfico. Pasos para una nueva cultura de la movilidad urbana. Madrid: Ministerio de Fomento.

Skinner, David; Rose, Paul (2007) Hell is other Cyclist" in Cycling and Society. Ashgate eBook. London

Spinney, Justin (2007) "Cycling the City:Non place and the sensority construction of Meaning in a Mobile practice" in Cycling and Society. Ashgate eBook. London

Villasante, Tomás R. (2004) Desbordes creativos, estilos y estrategias para la transformación social. Madrid: Catarata. 